\title{
Isoperimetric profile and random walks on locally compact solvable groups
}

\author{
Romain Tessera
}

\begin{abstract}
We study the large-scale geometry of a large class of amenable locally compact groups comprising all solvable algebraic groups over a local field and their discrete subgroups. We show that the isoperimetric profile of these groups is in some sense optimal among amenable groups. We use this fact to compute the probability of return of symmetric random walks, and to derive various other geometric properties.
\end{abstract}

\section{Introduction}

Pursuing our study [31] of large-scale Sobolev inequalities on metric measure spaces, we introduce a notion of large-scale foliation and we prove that if the space $X$ is large-scale foliated by the space $Y$, and if $Y$ satisfies a Sobolev inequality at large scale, then so does $X$. In particular the $L^{p}$-isoperimetric profile of $Y$ grows faster than that of $X$. A special case is when $Y=H$ is a closed unimodular subgroup of a locally compact unimodular group $X=G$. We apply this general fact to a special class of amenable groups, namely geometrically elementary solvable groups (see the precise definition below). This class of amenable locally compact groups is stable under quasi-isometries, and contains all compactly generated unimodular closed subquotients of the group of upper triangular matrices $\mathrm{T}(d, k)$ over a finite product of local fields. If $G$ is a geometrically elementary solvable group with exponential growth, we prove that the $L^{p}$-isoperimetric profile of $G$ satisfies

$$
j_{G, p}(t) \approx \log t
$$

for every $1 \leq p \leq \infty$. As a consequence, the probability of return of a symmetric random walk on such a group decreases like

$$
\phi(n) \approx e^{-n^{1 / 3}} .
$$

Mathematics Subject Classification (2010): 20F65; 22E35.

Keywords: Solvable locally compact groups, isoperimetric profile, random walks on groups, $L^{p}$ cohomology, uniform embeddings into Banach spaces. 
Most results regarding (1.1) and (1.2) over the past 25 years have their source in the seminal work of Varopoulos ([32]-[40]).

To be more specific, our work generalizes and unifies older results, where (1.1) and (1.2) had been proved for unimodular amenable connected Lie groups and their lattices [2], [17], [24], [39] (see also the expository paper [26]), and for some finitely generated groups such as the lamplighter or solvable Baumslag-Solitar's groups [9]. Other groups for which these behaviors have been established are finitely generated solvable torsion-free ${ }^{1}$ groups with finite Prüfer rank [25].

We prove a stronger statement when the group is either a quotient of a solvable algebraic group over a $q$-adic field ( $q$ is a prime), a closed subquotient of an amenable connected Lie group, or a cocompact lattice in a finite product of such groups (such as solvable Baumslag-Solitar groups). For these groups, the isoperimetric profile inside a ball grows linearly with the radius of the ball. This extends a previous result [29], where this had been shown for connected solvable Lie groups (and their lattices).

The rest of the introduction is devoted to a more comprehensive and precise exposition of the results, together with some of their applications. In more detail, after recalling a few definitions, we shall state our main results about the $L^{p}$-isoperimetric profile, and the $L^{p}$-isoperimetric profile in balls. Then we shall explain how these estimates for the $L^{2}$-isoperimetric profile can be used to provide sharp lower bounds on the probability of return of random walks. Our estimates on the $L^{p}$-isoperimetric profile in balls will then be applied to compute the $L^{p}$-compression of these groups, and to prove that their first reduced $L^{p}$-cohomology vanishes for all $1<p<\infty$ (confirming a conjecture of Gromov). We then give a few interesting examples of groups in the class GES. We close the introduction with a short list of open problems.

Notation and basic definitions. Let $G$ be a locally compact, compactly generated group equipped with a left Haar measure $\mu$. Let $S$ be a compact symmetric generating subset of $G$, i.e., $\cup_{n \in \mathbb{N}} S^{n}=G$. Equip $G$ with the left-invariant word metric associated to $S$, i.e., $d_{S}(g, h)=\inf \left\{n, g^{-1} h \in S^{n}\right\}$. The closed ball of center $g$ and of radius $r$ is denoted by $B(g, r)$ and its volume by $V(r)$. Let $\lambda$ be the action of $G$ by left translations on functions on $G$, i.e., $\lambda(g) f(x)=f\left(g^{-1} x\right)$. Restricted to elements of $L^{p}(G), \lambda$ is called the left regular representation of $G$ on $L^{p}(G)$.

For any $1 \leq p \leq \infty$, and any subset $A$ of $G$, the $L^{p}$-isoperimetric profile inside $A$ is denoted by

$$
J_{p}(A)=\sup _{f \in L^{p}(A)} \frac{\|f\|_{p}}{\sup _{s \in S}\|f-\lambda(s) f\|_{p}}
$$

where $L^{p}(A)$ comprises all elements $L^{p}(G)$ essentially supported in $A$.

\footnotetext{
${ }^{1}$ In fact, in [25] the authors claim to prove this for all finitely generated solvable groups with finite Prüfer rank, but their proof is based on the wrong idea (last statement of Proposition 4.1 in [25]) that the torsion subgroup of the nilpotent radical of a finite Prüfer rank solvable group is finite (cf. Hall's example mentioned at the end of this introduction).
} 
Isoperimetric profile. (see for instance [7]) The $L^{p}$-isoperimetric profile, is the nondecreasing function

$$
j_{G, p}(v)=\sup _{\mu(A) \leq v} J_{p}(A)
$$

Isoperimetric profile inside balls. [29] The $L^{p}$-isoperimetric profile inside balls is the nondecreasing function

$$
J_{G, p}^{b}(r)=J_{p}(B(1, r)) .
$$

We will be interested in the "asymptotic behavior" of these functions which we now define. Let $f, g: \mathbb{R}_{+} \rightarrow \mathbb{R}_{+}$be two monotonic functions. We write $f \preceq g$, if there exists $C>0$ such that $f(t)=O(g(C t))$ when $t \rightarrow \infty$. We write $f \approx g$ if both $f \preceq g$ and $g \preceq f$. The asymptotic behavior of $f$ is its class modulo the equivalence relation $\approx$.

\section{Main results.}

Theorem 1 (see Corollary 4.3). Let $G$ and $H$ be compactly generated unimodular groups, such that $H$ is either a closed subgroup or a quotient of $G$. Let $1 \leq p \leq \infty$. Then,

$$
j_{G, p} \preceq j_{H, p}
$$

If moreover $H$ is not distorted in $G$, then

$$
J_{G, p}^{b} \preceq J_{H, p}^{b} .
$$

If the groups are finitely generated, these statements are much easier to prove (see [14]).

Remark 1.1. If $H$ is a quotient, then the second statement does not require that the groups are unimodular.

We also show:

Proposition 2 (see Propositions 4.5, 4.6 and 4.7). Let $1 \rightarrow H \rightarrow G \rightarrow Q \rightarrow 1$ be an exact sequence of compactly generated locally compact groups. Let $1 \leq p \leq \infty$. Then, if $H$ is compact, then $j_{G, p} \approx j_{Q, p}$, and $J_{G, p}^{b} \approx J_{Q, p}^{b}$. If instead $Q$ is compact, then $j_{G, p} \approx j_{H, p}$, and $J_{G, p}^{b} \approx J_{H, p}^{b}$.

According to a theorem of Coulhon and Saloff-Coste [11], if $G$ is a compactly generated, locally compact group with exponential growth, then $j_{G, p}(t) \preceq \log t$. On the other hand it is very easy to see that $J_{G, p}(t) \leq 2 t$.

We will now combine these stability results with the following central result of [31] in order to define a large class of groups satisfying the converse inequalities.

Theorem 3. Let $(G, S)$ and $(H, T)$ be two compactly generated, locally compact groups, equipped with symmetric generating subsets $S$ and $T$ respectively. Then, the asymptotic behavior of $j_{G, p}, J_{G, p}^{b}$, for any $1 \leq p \leq \infty$ does not depend on $S$. Moreover, if $G$ and $H$ are both unimodular, and if $G$ is quasi-isometric to $H$, then

$$
j_{G, p} \approx j_{H, p} \text { and } J_{G, p}^{b} \approx J_{H, p}^{b} .
$$


Geometrically Elementary Solvable groups. The class GES of geometrically elementary solvable groups is the smallest class of (compactly generated) locally compact groups

- comprising all unimodular closed compactly generated subgroups of the group $\mathrm{T}(d, k)$, for any integer $d$ and any local field $k$;

- stable under taking finite (direct) products, quotients, and unimodular closed compactly generated subgroups;

- stable under quasi-isometry.

As we will see below, this class contains as particular examples solvable Baumslag-Solitar groups, lamplighter groups, and polycyclic groups. Let us now state our main result about this class.

Theorem 4. Let $G$ be a GES group. Then, for every $1 \leq p \leq \infty$,

$$
j_{G, p}(t) \succeq \log t
$$

This result was already known for polycylic groups [23], connected amenable Lie groups [24], and for the lamplighter and other particular examples [9]. To prove Theorem 4, we establish a stronger result for the group of triangular matrices $\mathrm{T}(d, k)$ over a local field $k$, i.e., that $J_{G, p}^{b}(t) \succeq t$. Indeed, note that $J_{G, p}(r) \leq$ $j_{G, p}(V(r))$. So, in particular, if the group has exponential growth, $J_{G, p}^{b}(t) \succeq t$ implies $j_{G, p} \succeq \log t$. The stability under finite product being trivial, we obtain all geometric elementary solvable groups using Theorems 1, 2 and 3.

Restricting to groups with exponential growth, we obtain:

Corollary 5. Let $G$ be a geometrically elementary solvable group with exponential growth. Then, for every $1 \leq p \leq \infty$,

$$
j_{G, p}(t) \approx \log t
$$

It is natural to ask whether a geometrically elementary solvable group satisfies the stronger property $J_{G, p}^{b}(t) \succeq t$. Since its variable is the radius of a ball, $J_{G, p}^{b}$ is sensitive to distortion of the metric. Note that this is reflected in the statement of Theorem 1.

Theorem 6. Let $q$ be a prime and let $k$ be a q-adic field. Let $G$ be either a quotient of a compactly generated algebraic group over $k$, or a subquotient of a closed compactly generated subgroup of an almost connected amenable Lie group. Then, for every $1 \leq p \leq \infty$,

$$
J_{G, p}^{b}(t) \approx t
$$

In particular these groups have controlled Følner sequences (see [30]).

In [29], we proved this statement for connected amenable Lie groups, lamplighter groups, and solvable Baumslag-Solitar groups. These are not special cases of Theorem 6 , but will be of Theorem 5.3 (which is a little too technical to state in this introduction). 
Application to random walks. Let $G$ be a locally compact, compactly generated group. Using Theorem 9.2 of [31] (which is a straightforward generalization of Theorem 7.1 in [7]), we obtain the following result.

Theorem 7 (see Theorem 6.2). Let $G$ be a geometrically elementary solvable group with exponential growth, and let $\mu$ be a symmetric probability measure whose support is compact and generates $G$. Then, for every compact neighborhood $U$ of the neutral element,

$$
\mu^{2 n}(U) \approx e^{-n^{1 / 3}}
$$

As already mentioned, this fact was known for connected unimodular amenable Lie groups [24], for finitely generated torsion-free solvable groups with finite Prüfer rank [25], and for the lamplighter group $F(\mathbb{Z}$, where $F$ is a finite group in [8]. Using a probabilistic approach, Mustapha [22] was able to prove it for certain $p$-adic analytic unimodular groups (which are particular cases of geometrically elementary solvable groups).

\section{Application to $L^{p}$-compression.}

Equivariant $\boldsymbol{L}^{p}$-compression. Recall that the equivariant $L^{p}$-compression rate $B_{p}(G)$ of a locally compact compactly generated group is the supremum of $0 \leq$ $\alpha \leq 1$ such that there exists a proper isometric affine action $\sigma$ on some $L^{p}$-space satisfying, for all $g \in G$,

$$
\|\sigma(g) .0\|_{p} \geq C^{-1}|g|_{S}^{\alpha}-C
$$

for some constant $C<\infty,|g|_{S}$ being the word length of $g$ with respect to a compact generating subset $S$.

It follows from Corollary 13 of [29] that a group with linear $L^{p}$-isoperimetric profile inside balls satisfies $B_{p}(G)=1$. Hence, we obtain:

Theorem 8. The groups appearing in Theorem 6 (or more generally Theorem 5.3) satisfy $B_{p}(G)=1$ for any $1 \leq p \leq \infty$.

Nonequivariant $\boldsymbol{L}^{p}$-compression. Recall that the $L^{p}$-compression rate of a metric space $(X, d)$ is the supremum of all $0 \leq \alpha \leq 1$ such that there exists a map $F$ from $X$ to some $L^{p}$-space satisfying, for all $x, y \in X$,

$$
C^{-1} d(x, y)^{\alpha}-C \leq\|F(x)-F(y)\|_{p} \leq d(x, y),
$$

for some constant $C<\infty$.

Another theorem of Mustapha [21] says that an algebraic compactly generated subgroup of $\mathrm{GL}(d, k)$, where $k$ is a $q$-adic field, is non distorted in $\mathrm{GL}(d, k)$. As $\mathrm{T}(d, k)$ is cocompact in $\mathrm{GL}(d, k)$ and satisfies $B_{p}(\mathrm{~T}(d, k))=1$, we obtain:

Theorem 9. Let $k$ be a q-adic field. Let $G$ be an algebraic compactly generated subgroup of $G L(d, k)$. Then, the $L^{p}$-compression rate of $G$ satisfies $R_{p}(G)=1$ for any $1 \leq p \leq \infty$. 
Application to the first reduced $\boldsymbol{L}^{p}$-cohomology. Recall that a conjecture of Gromov [15] states that all amenable discrete groups have trivial reduced $L^{p}$-cohomology, in degree at least 1 , and for any $1<p<\infty$. Cheeger and Gromov proved it when $p=2[8]$. The question is obviously relevant in the more general setting of locally compact groups. In [30], we were able to settle the conjecture in degree 1 for the class of amenable groups with linear isoperimetric profile in balls. The present paper therefore extends the class of groups satisfying Gromov's conjecture in degree 1.

Theorem 10. Let $G$ be as in Theorem 6 (or more generally Theorem 5.3). Then for every $1<p<\infty, \bar{H}^{1}\left(G, \lambda_{G, p}\right)=0$.

Some remarks about the class GES. As solvable connected Lie groups embed as closed subgroups of $\mathrm{T}(d, \mathbf{C})$, we deduce that unimodular amenable connected Lie groups, hence polycyclic groups are in GES (see the discussion at the beginning of Section 5).

The class GES contains the solvable Baumslag-Solitar groups BS $(1, n)=\langle t, x|$ $\left.t x t^{-1}=x^{n}\right\rangle$, for any integer $n$ with $|n| \geq 1$. This group can be described as $\operatorname{BS}(1, n)=\mathbb{Z}[1 / n] \rtimes \mathbb{Z}$, where $\mathbb{Z}$ acts on $\mathbb{Z}[1 / n]$ by multiplication by $n$. It has a faithful matrix representation

$$
\mathrm{BS}(1, n)=\left\{\left(\begin{array}{cc}
n^{k} & P \\
0 & 1
\end{array}\right), k \in \mathbb{Z}, P \in \mathbb{Z}[1 / n]\right\} .
$$

Let $\mathbb{Q}_{p}$ denote the $p$-adic field, and define the ring $\mathbb{Q}_{n}$ as the direct product of all $\mathbb{Q}_{p}$ when $p$ ranges over the set of distinct prime divisors of $n$. Then the natural diagonal embedding of $\mathbb{Z}[1 / n]$ into $\mathbb{Q}_{n} \oplus \mathbb{R}$ has discrete and actually cocompact image (see Chap. IV, $\S 2$ in [41]). Accordingly, $\mathrm{BS}(1, n)$ can be seen as a cocompact lattice inside the group $\left(\mathbb{Q}_{n} \oplus \mathbb{R}\right) \rtimes \mathbb{Z}$, where $\mathbb{Z}$ acts (diagonally) by multiplication by powers of $n$.

Similarly if $p$ is a prime integer, the lamplighter group $\mathbb{F}_{p} \succ \mathbb{Z}=\left(\oplus_{\mathbb{Z}} \mathbb{F}_{p}\right) \rtimes \mathbb{Z}$ admits a faithful matrix representation

$$
\mathbb{F}_{p}\left\{\mathbb{Z}=\left\{\left(\begin{array}{cc}
X^{k} & P \\
0 & 1
\end{array}\right), k \in \mathbb{Z}, P \in \mathbb{F}_{p}\left[X, X^{-1}\right]\right\},\right.
$$

and sits as a cocompact lattice inside $\mathbb{F}_{p}((X)) \oplus \mathbb{F}_{p}\left(\left(X^{-1}\right)\right) \rtimes \mathbb{Z}$, where $\mathbb{Z}$ acts by multiplication by powers of $X$.

Note that the class GES also contains groups which are not virtually solvable. Indeed the lamplighter group $F \imath \mathbb{Z}=F^{(\mathbb{Z})} \rtimes \mathbb{Z}$, where $F$ is any finite group belongs to GES. Namely, such a group is quasi-isometric to any $F^{\prime} \backslash \mathbb{Z}$ where $F^{\prime}$ has same cardinality as $F$, [13]. Hence one can take $F^{\prime}$ to be a product of $\mathbb{F}_{q}$ for finitely many primes $q$, which then implies that $F^{\prime} \imath \mathbb{Z}$ is a subgroup in a finite product of lamplighter groups $\mathbb{F}_{q}<\mathbb{Z}$.

Finally, let us mention that the class GES contains finitely generated groups which are not residually finite (hence not linear) as shown by the following example 
due to Hall, [16]. Fix a prime $q$ and consider the group of upper triangular 3 by 3 matrices:

$$
G=\left\{\left(\begin{array}{ccc}
1 & x & z \\
0 & q^{n} & y \\
0 & 0 & 1
\end{array}\right) ; \quad x, y, z \in Z[1 / q] ; n \in \mathbb{Z}\right\} .
$$

Taking the quotient by the central infinite cyclic subgroup of unipotent matrices $I+m E_{1,3}$ where $m \in \mathbb{Z}$, we obtain an elementary solvable group which is nonresidually finite since its center is isomorphic to $\mathbb{Z}[1 / q] / \mathbb{Z}$.

\section{Open problems.}

Conjecture 1.2. We conjecture that all GES groups and all compactly generated closed subgroups of triangulable groups over a product of local fields satisfy $J_{G, p}(t) \approx t$ (to prove the last statement, one needs to study the distortion of such subgroups).

Question 1.3. Are the classes of groups satisfying $j_{G, p} \approx \log t$, resp. $J_{G, p}(t) \approx t$, stable under extension?

Question 1.4. Conversely, does every group satisfying $j_{G, p} \succeq \log t$ belong to the class GES?

Organization of the paper. In Section 2, we briefly recall the notions of Sobolev inequalities at scale $h$ and the results of [31] that we need here. We also discuss some subtleties occurring when the group is not unimodular. In Section 3, we prove our main technical result about large-scale foliations of metric measure spaces. This is the main ingredient of the proof of Theorem 1. In Section 4, we work out the case of closed subgroups and quotients. In Section 5, we prove Theorems 4 and 6. Finally, in Section 6, we prove Theorem 7.

Acknowledgments. I am grateful to Yves de Cornulier for pointing out to me the group constructed by Hall [16], and for his useful remarks and corrections. I would also like to thank the referee for pointing out missing references.

\section{Preliminaries: functional analysis at a given scale}

The purpose of this section is to briefly recall the notions introduced in [31]. By a metric measure space $(X, d, \mu)$ we mean a metric space $(X, d)$ equipped with a Borel measure $\mu$ on $X$ such that bounded measurable subsets have finite measure. The volume of the closed ball $B_{X}(x, r)$ is denoted by $V_{X}(x, r)$ (or simply $V(x, r)$ if only one space is involved).

\subsection{The locally doubling property}

The metric measure spaces that we will consider satisfy a very weak property of bounded geometry introduced in [10] in the context of Riemannian manifolds. 
Definition 2.1. We say ${ }^{2}$ that a space $X$ is locally doubling at scale $r>0$ if there exists a constant $C_{r}$ such that

$$
V(x, 2 r) \leq C_{r} V(x, r) \quad \forall x \in X
$$

If it is locally doubling at every scale $r>0$, then we just say that $X$ is locally doubling.

Example 2.2. Let $X$ be the vertex set of a connected graph with degree bounded by $d$, equipped with the counting measure. The volume of balls of radius $r$ satisfies

$$
1 \leq V(x, r) \leq d^{r} \quad \forall x \in X
$$

In particular, $X$ is locally doubling.

Example 2.3. Let $(X, d, \mu)$ be a metric measure space and let $G$ be a locally compact group acting by measure-preserving isometries. If $G$ acts cocompactly, then $X$ is locally doubling.

\subsection{Local norm of the gradient at scale $h$}

The purpose of this section is to define a notion of "local norm of the gradient" (whose infinitesimal analogue is the modulus of the gradient of a smooth function on a Riemannian manifold), which captures the local variations of a function defined on a metric measure space $(X, d, \mu)$.

For every $h>0$, we define an operator $|\nabla|_{h}$ on $L^{\infty}(X)$ by

$$
|\nabla f|_{h}(x)=\sup \{|f(y)-f(x)|, d(x, y) \leq h\}, \quad \forall f \in L^{\infty}(X) .
$$

\subsection{Sobolev inequalities}

Let $\varphi: \mathbb{R}_{+} \rightarrow \mathbb{R}_{+}$be an increasing function and let $p \in[1, \infty]$. The following formulation of Sobolev inequality was first introduced in [6] in the context of Riemannian manifolds.

Definition 2.4. One says that $X$ satisfies a Sobolev inequality $\left(S_{\varphi}^{p}\right)$ at scale at least $h$ if there exist $C, C^{\prime}>0$ such that

$$
\|f\|_{p} \leq C \varphi\left(C^{\prime}|\Omega|\right)\left\||\nabla f|_{h}\right\|_{p}
$$

where $\Omega$ ranges over all compact subsets of $X,|\Omega|$ denotes the measure $\mu(\Omega)$, and $f \in L^{\infty}(\Omega), L^{\infty}(\Omega)$ being the set of elements of $L^{\infty}(X)$ with support in $\Omega$. We say that $X$ satisfies a large-scale Sobolev inequality $\left(S_{\varphi}^{p}\right)$ if it satisfies a Sobolev inequality at scale $h$ for $h$ large enough.

\footnotetext{
${ }^{2} \operatorname{In}[10]$ and in [28], the local doubling property is denoted (DV) loc.
} 


\subsection{Isoperimetric profiles}

Let $A$ be a measurable subset of $X$, and let $h>0$. We define

$$
J_{h, p}(A)=\sup _{f} \frac{\|f\|_{p}}{\left\||\nabla f|_{h}\right\|_{p}},
$$

where the supremum is taken over functions $f \in L^{\infty}(A)$.

Definition 2.5 ([31]). The $L^{p}$-isoperimetric profile $j_{X, h, p}$ (respectively, inside balls: $\left.J_{X, h, p}^{b}\right)$ is a nondecreasing function defined by

$$
j_{X, h, p}(v)=\sup _{|A| \leq v} J_{h, p}(A) .
$$

(respectively, $J_{X, h, p}^{b}(t)=\sup _{x \in X} J_{h, p}(B(x, t))$.) In the sequel, we will generally omit the scale, and only denote $j_{X, p}$ instead of $j_{X, h, p}$.

Remark 2.6. One can check that $j_{X, p} \preceq j_{X, q}$ is always true when $p \leq q<\infty$. Moreover, in most cases (e.g., all known examples of groups), $j_{X, p} \approx j_{X, q}$.

\subsection{Link between Sobolev inequalities and isoperimetric profiles}

Sobolev inequalities $\left(S_{\varphi}^{p}\right)$ can also be interpreted as $L^{p}$-isoperimetric inequalities. Clearly, the space $X$ always satisfies the Sobolev inequality $\left(S_{\varphi}^{p}\right)$ with $\varphi=j_{X, p}$. Conversely, if $X$ satisfies $\left(S_{\varphi}^{p}\right)$ for a function $\varphi$, then

$$
j_{X, p} \succeq \varphi .
$$

\section{6. $L^{2}$-profile and probability of return of random walks}

The case $p=2$ is of particular interest as it contains some probabilistic information on the space $X$. Indeed, it was shown in [8] that for manifolds with bounded geometry, there is a good correspondence between upper bounds of the largetime on-diagonal behavior of the heat kernel and Sobolev inequality $\left(S_{\varphi}^{2}\right)$. In the survey [7], a similar statement is proved for the standard random walk on a weighted graph. In Theorem 9.1 of [31], we give a discrete-time version of this theorem for general metric measure spaces.

Let $(X, d, \mu)$ be a metric measure space. Consider a measurable family of probability measures $P=\left(P_{x}\right)_{x \in X}$ such that the operator on $L^{2}(X, \mu)$ defined by $\operatorname{Pf}(x)=\int_{X} f(y) d P_{x}(y)$ is self-adjoint. This is equivalent to saying that the random walk with transition probabilities $P=\left(P_{x}\right)_{x \in X}$ is reversible with respect to the measure $\mu$. We will also ask $P$ to focus on the geometry at scale $h>0$ in the following sense: there exist a "large" constant $1 \leq A<\infty$ and a "small" constant $c>0$ such that for ( $\mu$-almost) every $x \in X$ :

(i) $P_{x} \ll \mu$;

(ii) $p_{x}=d P_{x} / d \mu$ is supported in $B(x, A h)$;

(iii) $p_{x}$ is larger than $c$ on $B(x, h)$. 
We will need the following particular case of Theorem 9.2 in [31]:

Theorem 2.7. Let $X=(X, d, \mu)$ be a metric measure space. Then, the large-scale isoperimetric profile satisfies

$$
j_{X, 2}(t) \approx \log t
$$

if and only if, for any reversible random walk at a large enough scale, we have

$$
\sup _{x \in X} p_{x}^{2 n}(x) \approx e^{-n^{1 / 3}} \quad \forall n \in \mathbb{N} .
$$

\subsection{Large-scale equivalence between metric measure spaces}

Definition 2.8. Let $(X, d, \mu)$ and $\left(X^{\prime}, d^{\prime}, \mu\right)$ two spaces satisfying the locally doubling property. Let us say that $X$ and $X^{\prime}$ are large-scale equivalent if there is a function $F$ from $X$ to $X^{\prime}$ with the following properties:

(a) There exist two unbounded increasing functions $\rho_{1}, \rho_{2}: \mathbb{R}_{+} \rightarrow \mathbb{R}_{+}$such that for all $x, y \in X$,

$$
\rho_{1}\left(d_{X}(x, y)\right) \leq d_{X^{\prime}}(F(x), F(y)) \leq \rho_{2}\left(d_{X}(x, y)\right) .
$$

(b) $F$ is almost onto, i.e., there exists a constant $C$ such that $[F(X)]_{C}=X^{\prime}$.

(c) For $r>0$ large enough, there is a constant $C_{r}>0$ such that for all $x \in X$

$$
C_{r}^{-1} V_{X}(x, r) \leq V_{X^{\prime}}(F(x), r) \leq C V_{X}(x, r) .
$$

Remark 2.9. Note that being large-scale equivalent is an equivalence relation between metric measure spaces with the locally doubling property.

Remark 2.10. If $X$ and $X^{\prime}$ are quasi-geodesic, then (a) and (b) imply that $F$ is roughly bi-Lipschitz: there exists $C \geq 1$ such that

$$
C^{-1} d(x, y)-C \leq d(F(x), F(y)) \leq C d(x, y)+C .
$$

In this case, (a) and (b) correspond to the classical definition of a quasi-isometry.

Example 2.11 ([31]). Consider the subclass of metric measure spaces including graphs with bounded degree, equipped with the countable measure; Riemannian manifolds with bounded geometry ${ }^{3}$, equipped with the Riemannian measure; compactly generated, locally compact groups equipped with a left Haar measure and a word metric associated to a compact, generating subset. In this class, quasiisometries are always large-scale equivalences.

The following theorem generalizes [10], which was established in the context of graphs and Riemannian manifolds with bounded geometry.

\footnotetext{
${ }^{3}$ Classically, this means: with Ricci curvature bounded from below, and with bounded radius of injectivity. These assumptions are generally used in order to perform some discretization of functional inequalities (see for instance [19]).
} 
Theorem 2.12 (Theorem 8.1 in [31]). Let $F: X \rightarrow X^{\prime}$ be a large-scale equivalence between two spaces $X$ and $X^{\prime}$ satisfying the locally doubling property. Assume that for $h>0$ fixed, the space $X$ satisfies a Sobolev inequality $\left(S_{\varphi}^{p}\right)$ at scale $h$, then there exists $h^{\prime}$, depending only on $h$ and on the constants of $F$ such that $X^{\prime}$ satisfies $\left(S_{\varphi}^{p}\right)$ at scale $h^{\prime}$. In particular, large-scale Sobolev inequalities are invariant under large scale equivalence.

\section{Large-scale foliation of a metric measure space and mono- tonicity of the isoperimetric profile}

Definition 3.1. Let $X=\left(X, d_{X}, \mu\right)$ and $Y=\left(Y, d_{Y}, \lambda\right)$ be two metric measure spaces satisfying the locally doubling property. We say that $X$ is large-scale foliated (respectively, normally large-scale foliated) by $Y$ if it admits a measurable partition $X=\sqcup_{z \in Z} Y_{z}$ satisfying the first two (respectively, three) of the following conditions:

- (measure decomposition) There exists a measure $\nu$ on $Z$ and a measure $\lambda_{z}$ on $\nu$-almost every $Y_{z}$ such that for every continuous compactly supported function $f$ on $X$,

$$
\int_{X} f(x) d \mu(x)=\int_{Z}\left(\int_{Y_{z}} f(t) d \lambda_{z}(t)\right) d \nu(z) .
$$

The subsets $Y_{z}$ are called the leaves, and the space $Z$ is called the base of the foliation.

- (large-scale control) For $\nu$-almost every $z$ in $Z$, there exists $\alpha_{z}>0$ and a large scale equivalence $h_{z}:\left(Y, d_{Y}, \lambda\right) \rightarrow\left(Y_{z}, d_{X}, \alpha_{z} \nu_{z}\right)$, which is uniform with respect to $z \in Z$.

- (measure normalization) For all $r>0$, there exists $C_{r} \geq 1$ such that for all $z \in Z$ and all $x \in Y_{z}$,

$$
C_{r}^{-1} V_{Y_{z}}(x, r) \leq V_{X}(x, r) \leq C_{r} V_{Y_{z}}(x, r) .
$$

In particular, we can take $\alpha_{z}=1$.

Recall that the compression of a map $F$ between two metric space $X$ and $Y$ is the function $\rho$ defined by

$$
\rho(t)=\inf _{d_{X}\left(x, x^{\prime}\right) \geq t} d_{Y}\left(F(x), F\left(x^{\prime}\right)\right), \quad \forall t>0 .
$$

Definition 3.2. We call the compression of a large-scale foliation of $X$ by $Y$ the function

$$
\rho(t)=\inf _{z \in Z} \rho_{z}(t)
$$

where $\rho_{z}$ is the compression function of the large-scale equivalence $h_{z}$. 
A crucial example that we will consider in some details in the next section is the case where $Y=H$ is a closed subgroup of a locally compact group $G=X$ such that $G / H$ carries a $G$-invariant measure.

Theorem 3.3. Let $X=(X, d, \mu)$ and $Y=(Y, \delta, \lambda)$ be two metric measure spaces satisfying the locally doubling property. Assume that $X$ is normally large-scale foliated by $Y$. Then if $Y$ satisfies a Sobolev inequality $\left(S_{\varphi}^{p}\right)$ at scale $h$, then $X$ satisfies $\left(S_{\varphi}^{p}\right)$ at scale $h^{\prime}$, for $h^{\prime}$ large enough. In other words, if $j_{X, p}$ and $j_{Y, p}$ denote respectively the $L^{p}$-isoperimetric profiles of $X$ and $Y$ at the scales $h$ and $h^{\prime}$, then

$$
j_{Y, p} \succeq j_{X, p} .
$$

Moreover, if $\rho$ is the compression of the large-scale equivalence, then

$$
J_{Y, p}^{b} \succeq J_{X, p}^{b} \circ \rho .
$$

The last result is true under the weaker assumption that $X$ is merely large-scale foliated by $Y$.

The main difficulty comes from the fact that we need to control the measure of the support of the restriction to a leaf of a function defined on $X$. On the contrary, the control on the diameter of the support follows trivially from the definition of $\rho$ (and does not require the normalization condition). Hence the second inequality $\left(J_{Y, p}^{b} \succeq J_{X, p}^{b} \circ \rho\right)$ is much easier than the first one, and therefore left to the reader.

Definition 3.4. A subset $A$ of a metric space is called $h$-thick if it is a union of closed balls of radius $h$.

The following lemma implies that we can restrict to functions with thick support.

Lemma 3.5 (Proposition 8.3 in [31]). Let $X=(X, d, \mu)$ be a metric measure space. Fix some $h>0$ and some $p \in[1, \infty]$. There exists a constant $C>0$ such that for any $f \in L^{\infty}(X)$, there is a function $\tilde{f} \in L^{\infty}(X)$ whose support is included in an $h / 2$-thick subset $\Omega$ such that

$$
\mu(\Omega) \leq \mu(\operatorname{Supp}(f))+C
$$

and for every $p \in[1, \infty]$,

$$
\frac{\left\||\nabla \tilde{f}|_{h / 2}\right\|_{p}}{\|\tilde{f}\|_{p}} \leq C \frac{\left\||\nabla f|_{h}\right\|_{p}}{\|f\|_{p}} .
$$

On the other hand, the locally doubling property "extends" to thick subsets in the following sense:

Lemma 3.6. Let $X$ be a metric measure space satisfying the locally doubling property. Fix two positive numbers $u$ and $v$. There exists a constant $C=C(u, v)<\infty$ such that for any $u$-thick subset $A \subset X$, we have

$$
\mu\left([A]_{v}\right) \leq C \mu(A),
$$

where $[A]_{v}:=\{x \in X, d(x, A) \leq v\}$. 
Proof. Since $A$ is $u$-thick, it can be written as a union $\bigcup_{i \in I} B\left(x_{i}, u\right)$. Take a maximal subset $J \subset I$ such that the balls $B\left(x_{j}, u\right)$, with $j \in J$ are disjoint. By maximality of $J, A \subset[A]_{v} \subset \cup_{j} B\left(x_{j}, 4 u+v\right)$. Indeed, let $x \in[A]_{v}$, then the ball $B(x, v+3 u)$ contains a ball $B\left(x_{i}, u\right)$ for some $i \in I$. Therefore maximality implies that this ball must meet $B\left(x_{j}, u\right)$ for some $j \in J$, which implies the statement. The lemma now follows from the locally doubling condition.

The following statement says that large-scale equivalences roughly preserve the volume of big sets.

Lemma 3.7 (Proposition $8.5(2)$ in [31]). Let $X=(X, d, \mu)$ and $X^{\prime}=\left(X^{\prime}, d^{\prime}, \mu^{\prime}\right)$ be two spaces satisfying the locally doubling property. Let $F: X \rightarrow X^{\prime}$ be a largescale equivalence. Then there exists $C \geq 1$ such that for all $A \subset X$,

$$
\mu(A) \leq C \mu^{\prime}\left([F(A)]_{1}\right) .
$$

Finally, we will need:

Lemma 3.8. Assume that $X$ is normally large-scale foliated by $Y$. For a.e. $z \in Z$, let $\left[Y_{z}\right]_{1}$ be the 1-neighborhood of $Y_{z}$ in $X$. The inclusion map $\left(Y_{z}, d_{X}, \lambda_{z}\right) \rightarrow$ $\left(\left[Y_{z}\right]_{1}, d_{X}, \mu\right)$ is a large-scale equivalence, uniformly with respect to $z$.

Proof of Lemma 3.8. The two metric conditions (a) and (b) for being a largescale equivalence (see Definition 2.8) are trivially satisfied here. It remains to compare the volume of balls of fixed radius, which follows from the third condition of Definition 3.1, and from the fact that $X$ is doubling.

Proof of Theorem 3.3. Throughout the proof, we will use the letter $C$ as a generic constant, which might possibly take different values. Assume that $Y$ satisfies the Sobolev inequality $\left(S_{\varphi}^{p}\right)$. Let $\Omega$ be a compact subset of $X$ and $f \in L^{\infty}(\Omega)$. We want to prove that there exists $h^{\prime}$, depending only on $h$ and the spaces such that $f$ satisfies $\left(S_{\varphi}^{p}\right)$ at scale $h^{\prime}$. By Lemma 3.5 , we can assume that $\Omega$ is 1 -thick. For every $z \in Z$, denote by $f_{z}$ the restriction of $f$ to $Y_{z}$ and $\Omega_{z}=\Omega \cap Y_{z}$.

Claim 3.9. There exists $C<\infty$ such that, for every $z \in Z, \lambda_{z}\left(\Omega_{z}\right) \leq C \mu(\Omega)$.

Proof. Lemmas 3.8 and 3.7 imply that $\lambda_{z}\left(\Omega_{z}\right)$ is less than a constant times $\mu\left(\left[\Omega_{z}\right]_{1}\right)$, which is obviously less than $\mu\left([\Omega]_{1}\right)$, which by Lemma 3.6 is less than a constant times $\mu(\Omega)$.

By Theorem 2.12, there exists $h^{\prime}>0$ such that $Y_{z}$ satisfies $\left(S_{\varphi}^{p}\right)$ at scale $h^{\prime}$, uniformly with respect to $z \in Z$. So for every $z \in Z$,

$$
\left\|f_{z}\right\|_{p} \leq C \varphi\left(C \lambda_{z}\left(\Omega_{z}\right)\right)\left\|\left|\nabla f_{z}\right|_{h^{\prime}}\right\|_{p} .
$$

Since $\lambda_{z}\left(\Omega_{z}\right) \leq C \mu(\Omega)$ and $\varphi$ is nondecreasing, we have

$$
\left\|f_{z}\right\|_{p} \leq C \varphi(C \mu(\Omega))\left\|\left|\nabla f_{z}\right|_{h^{\prime}}\right\|_{p} .
$$

Moreover, we have

$$
\|f\|_{p}^{p}=\int_{Z}\left\|f_{z}\right\|_{p}^{p} d \nu(z) .
$$


Clearly, since $Y_{z}$ is equipped with the induced distance, for every $z \in Z$ and every $x \in Y_{z}$,

$$
|\nabla f|_{h^{\prime}}(x) \geq\left|\nabla f_{z}\right|_{h^{\prime}}(x) .
$$

Therefore,

$$
\left\||\nabla f|_{h^{\prime}}\right\|_{p}^{p} \geq \int_{Z}\left\|\left|\nabla f_{z}\right|_{h^{\prime}}\right\|_{p}^{p} d \nu(z)
$$

We then have

$$
\|f\|_{p} \leq C \varphi(C \mu(\Omega))\left\||\nabla f|_{h^{\prime}}\right\|_{p}
$$

and we are done.

\section{Application to locally compact groups}

\subsection{The case of groups: left and right translations}

Let $G$ be a locally compact, compactly generated group, and let $S$ be a generating set. Let $g \in G$ and let $f \in L^{p}(G)$ for some $1 \leq p \leq \infty$. We have

$$
|\nabla f|_{1}(g)=\sup _{s \in S}|f(g s)-f(g)| .
$$

In other words, if $\rho$ is the action of $G$ by right translation on functions, i.e., $\rho(g) f(x)$ $=f(x g)$, the isoperimetric profiles are therefore given by

$$
j_{G, p}(m)=\sup _{|\operatorname{Supp}(f)| \leq m} \frac{\|f\|_{p}}{\left\|\sup _{s \in S}|f-\rho(s) f|\right\|_{p}},
$$

and

$$
J_{G, p}^{b}(r)=\sup _{\operatorname{Supp}(f) \subset B(1, r)} \frac{\|f\|_{p}}{\left\|\sup _{s \in S} \mid f-\rho(s) f\right\|_{p}} .
$$

Proposition 4.1. We have

$$
j_{G, p} \approx \sup _{|\operatorname{Supp}(f)| \leq m} \frac{\|f\|_{p}}{\sup _{s \in S}\|f-\rho(s) f\|_{p}},
$$

and

$$
J_{G, p}^{b}(r) \approx \sup _{\operatorname{Supp}(f) \subset B(1, r)} \frac{\|f\|_{p}}{\sup _{s \in S}\|f-\rho(s) f\|_{p}} .
$$

Proof. Let us prove it for $j_{G, p}$ (the proof for $J_{G, p}^{b}$ is similar). Note that

$$
\begin{aligned}
\left\|\frac{1}{|S|}\left(\int_{S}|f-\rho(s) f|^{p} d s\right)^{1 / p}\right\|_{p}^{p} & =\frac{1}{|S|} \int_{S}\|f-\rho(s) f \mid\|_{p}^{p} d s \\
& \leq \sup _{s \in S}\|f-\rho(s) f\|_{p}^{p} \leq\left\|\sup _{s \in S}|f-\rho(s) f|\right\|_{p}^{p},
\end{aligned}
$$


so we get

$$
\begin{aligned}
j_{G, p} & \leq \sup _{|\operatorname{Supp}(f)| \leq m} \frac{\|f\|_{p}}{\sup _{s \in S}\|f-\rho(s) f\|_{p}} \\
& \leq \sup _{|\operatorname{Supp}(f)| \leq m} \frac{\|f\|_{p}}{\left\|\frac{1}{|S|}\left(\int_{S}|f-\rho(s) f|^{p} d s\right)^{1 / p}\right\|_{p}} .
\end{aligned}
$$

However, in Section 7 of [31] it was proved that

$$
j_{G, p} \approx \sup _{|\operatorname{Supp}(f)| \leq m} \frac{\|f\|_{p}}{\left\|\frac{1}{|S|}\left(\int_{S}|f-\rho(s) f|^{p} d s\right)^{1 / p}\right\|_{p}}
$$

So combining (4.1) and (4.2) proves the proposition.

\section{Left or right?}

One may wonder why we chose to define the isoperimetric profiles with left translations in the introduction, since according to the previous proposition, it seems that the correct definition should be with right translations. Indeed, since the metric is left-invariant, we have for all $s \in S, d(g, g s)=1$, whereas in general $d\left(s^{-1} g, g\right)$ is not even bounded. Here are the reasons for this choice:

- If the group $G$ is unimodular, then the isoperimetric profiles are the same, whether we define them by left translations, or by right translations.

- Suppose that the group $G$ is non-unimodular. Then if we define the profiles with left translations and if the group is amenable, $j_{G, p}=\infty$. On the other hand, if we define them with right translations, then both $j_{G, p}$ and $J_{G, p}^{b}$ are bounded and therefore behave as for a non amenable group.

- Finally, if we define $J_{G, p}^{b}$ with left translations and if the group is amenable, then it is always a non bounded increasing function.

In conclusion, we see that if the group is non-unimodular, then the asymptotic behavior of $j_{G, p}$ does not contain any interesting information on the group, whereas $J_{G, p}^{b}$ is interesting only if it is defined with left translations.

In the following sections, we will not change our notation but rather indicate whether we consider a "left-profile" or a "right-profile" on $G$.

\subsection{Closed subgroups}

Proposition 4.2. Let $H$ be a closed compactly generated subgroup of a locally compact, compactly generated group $G$. Assume that the quotient $G / H$ carries a $G$-invariant Borel measure, then $G$ is normally large-scale foliated by $H$.

Proof. Let $\nu$ be a $G$-invariant $\sigma$-finite measure on the quotient $Z=G / H$. Since $\nu$ is $G$-invariant, up to normalization, one can assume that for every continuous compactly supported function $f$ on $G$,

$$
\int_{G} f(g) d \mu(x)=\int_{Z}\left(\int_{H} f(g h) d \lambda(h)\right) d \nu(g H) .
$$


We claim that the partition $G=\sqcup_{g H \in Z} g H$ satisfies the three conditions of Definition 2.8. Clearly, the first one follows from the above decomposition of $\mu$. For every $g \in G$, the left translation by $g$ is an isometry of $G$, so that all the left cosets of $H$ are isometric (we consider these cosets equipped with the distance induced from that of $G$ ). On the other hand, since $H$ is a closed subgroup, the inclusion map $H \rightarrow G$ is a uniform embedding, i.e., satisfies (a) of Definition 2.8. This proves the second condition. Finally, the last condition follows from the left-invariance of both $\nu$ and $\mu$. Namely, the left-invariance of $\nu$ implies that, for every $g \in G$, the mesure $\lambda_{g}$ on $g H$ is the image of $\lambda$ under the map $h \rightarrow g h$ from $H$ to $g H$.

In Lemma 4 of [14], it is proved that if $H$ is finitely generated subgroup of a finitely generated group $G$, then $j_{H} \preceq j_{G}$. Here is a generalization of this easy result.

Corollary 4.3. Let $H$ be a closed, compactly generated subgroup of $G$ and let $1 \leq p \leq \infty$. Assume that $G / H$ carries a $G$-invariant measure. Then,

- the right-profiles satisfy $j_{G, p} \preceq j_{H, p}$;

- a weaker conclusion holds for the right-profiles inside balls: $J_{G, p}^{b} \preceq J_{H, p}^{b} \circ \rho$, where $\rho$ is the compression of the injection $H \hookrightarrow G$.

Proof. This follows from Theorem 3.3 and Proposition 4.2.

Remark 4.4. Corollary 4.3 holds in particular when $G$ and $H$ are both unimodular. Actually this is the only interesting situation since, by Lemma 11.10 in [31], a non-unimodular group always has a bounded right-profile, i.e., $\left\||\nabla f|_{h}\right\|_{p} \geq c\|f\|_{p}$ for some $c>0$ depending only on $p \geq 1$ and $h \geq 1$. On the other hand, if $H$ is non-unimodular and if $G$ is unimodular and amenable, then, by Proposition 11.11 in [31] all the conclusions of Corollary 4.3 fall apart ${ }^{4}$.

\subsection{Quotients and cocompact subgroups}

Proposition 4.5. Let $Q=G / H$ be the quotient of a locally compact, compactly generated group $G$ by a closed normal subgroup $H$. Then for all $1 \leq p \leq \infty$, the left-profiles in balls satisfy $J_{G, p}^{b} \preceq J_{Q, p}^{b}$. Moreover if the groups are unimodular, then the left-profiles satisfy $j_{G, p} \preceq j_{Q, p}$.

Proof. We denote by $\pi$ the projection on $G / H$. Let us equip $G$ and $H$ with left Haar measures $\mu$ and $\nu$. Take a Haar measure $\lambda$ on $H$ such that for every continuous compactly supported function $f$ on $G$,

$$
\int_{G} f(g) d \mu(g)=\int_{Q}\left(\int_{H} f(g h) d \lambda(h)\right) d \nu(g H) .
$$

\footnotetext{
${ }^{4}$ For example, consider the non-unimodular group $H$ of positive affine transformations of $\mathbb{R}$ : this group, equipped with its left-invariant Riemannian metric is isometric to the hyperbolic plane. In particular, it has a bounded isoperimetric right-profile. On the other hand, it is a closed subgroup of the solvable unimodular Lie group Sol, whose isoperimetric profile $j_{G, p}$ is asymptotically equivalent to $\log t,[24]$.
} 
Let $S$ be a symmetric compact generating subset of $G$ and consider its image $T$ under the projection onto $Q$. The projection $\pi$ is therefore 1-Lipschitz between $(G, S)$ and $(Q, T)$. For every $1 \leq p<\infty$, consider the mapping $\Psi: C_{0}(G) \rightarrow C_{0}(Q)$ defined by

$$
\Psi(f)(g H)=\left(\int_{H}|f(g h)|^{p} d \lambda(h)\right)^{1 / p} .
$$

Moreover, $\Psi$ preserves the $L^{p}$-norm. Take $s \in S$ and $t=\pi(s), g \in G$ and $q=\pi(g)$. Then,

$$
\begin{aligned}
\left|\Psi(f)\left(t^{-1} q\right)-\Psi(f)(q)\right| & =\left(\int_{H}\left|f\left(s^{-1} g h\right)\right|^{p} d \lambda(h)\right)^{1 / p}-\left(\int_{H}|f(g h)|^{p} d \lambda(h)\right)^{1 / p} \\
& \leq\left(\int_{H}\left|f\left(s^{-1} g h\right)-f(g h)\right|^{p} d \lambda(h)\right)^{1 / p} .
\end{aligned}
$$

Therefore,

$$
\|\lambda(t) \Psi(f)-\Psi(f)\|_{p} \leq\|\lambda(s) f-f\|_{p} .
$$

Finally, observe that the support of $\Psi(f)$ is the projection of the support of $f$, so the first statement follows. Let us prove the second statement. By Lemma 3.5, we can suppose that the support of $f$ is 1-thick, so that the projection decreases volumes (up to a multiplicative constant).

Proposition 4.6. Let $Q=G / K$ be the quotient of a locally compact, compactly generated group $G$ by a closed normal compact subgroup $K$. Then, for all $1 \leq p \leq \infty$, the (left or right) profiles satisfy $j_{G, p} \approx j_{Q, p}$ and the (left or right) profiles in balls satisfy $J_{G, p}^{b} \approx J_{Q, p}^{b}$.

Proof. Note that by the previous proposition, we only need to show that $j_{G, p} \succeq$ $j_{Q, p}$ and $J_{G, p}^{b} \succeq J_{G, p}^{b}$. Consider compact generating sets $S$ and $\pi(S)$ in $G$ and $Q$, respectively, and suppose that the Haar measures on $G$ and $Q$ are such that $\mu\left(\pi^{-1}(A)\right)=\nu(A)$ for every Borel subset $A \subset Q$ with finite measure. Note that composition with $\pi$ defines a map $C_{0}(Q) \rightarrow C_{0}(G)$ which induces an isometry $\Phi: L^{p}(Q) \rightarrow L^{p}(G)$. On the other hand since $K$ is normal, one checks easily that $\Phi$ commutes with both the left and right regular representations. Indeed, $\lambda(s) \Phi(f)=\Phi(\lambda(\pi(s)) f$ ) (and the same for $\rho)$. Combining this with the fact that $\Phi$ is an isometry, yields the following equality: for all $s \in S$,

$$
\|\lambda(s) \Phi(f)-\Phi(f)\|_{p}=\|\lambda(\pi(s)) f-f\|_{p} .
$$

This proves the proposition.

Proposition 4.7. Let $1 \rightarrow H \rightarrow G \rightarrow Q \rightarrow 1$ be a short exact sequence of compactly generated locally compact groups such that $H$ is closed and $Q$ is compact. Then, for all $1 \leq p \leq \infty$, the profiles satisfy $j_{G, p} \approx j_{H, p}$, and the profiles in balls satisfy $J_{G, p}^{b} \approx J_{H, p}^{b}$. 
Proof. We only need to show the inequalities $j_{G, p} \succeq j_{H, p}$ and $J_{G, p}^{b} \succeq J_{G, p}^{b}$. Let $\eta: Q \rightarrow G$ be a measurable section such that $K=\eta(Q)$ is relatively compact. Each element $g \in G$ can be uniquely written as $\eta(q) h$, with $q \in Q$ and $h \in H$, and the left Haar measure on $G$ is identified (up to normalization) as the direct product $^{5}$ of the left Haar measures of $Q$ and $H$. Define an isometric embedding $\Phi: L^{p}(H) \rightarrow L^{p}(G)$ by

$$
\Phi(f)(k h)=f(h) .
$$

Suppose that the Haar measure of $Q$ has total mass equal to 1 . Then the measure of the support of $\Phi(f)$ equals the measure of the support of $f$. On the other hand, since the inclusion $H \rightarrow G$ is a quasi-isometry, the diameter of the support of $\phi(f)$ is less than a constant times the diameter of the support of $f$.

Finally let $S$ be a compact generating subset of $G$. For all $s \in S, h \in H, q \in Q$ and $f \in L^{p}(H)$, we have

$$
\Phi(f)(s \eta(q) h)-\Phi(f)(\eta(q) h)=\Phi(f)\left(\eta\left(q^{\prime}\right) t h\right)-\Phi(f)(\eta(q) h)=f(t h)-f(h),
$$

where $q^{\prime}=\bar{g} q \in Q$, and $t=\eta\left(q^{\prime}\right)^{-1} s \eta(q)$ is an element of $K^{-1} S K \cap H$ which is relatively compact in $H$. The proposition now follows trivially.

\section{Geometrically elementary solvable groups}

As already mentioned in the introduction, Theorems 4 and 6 will be consequences of the following facts:

- the stability results: Corollary 4.3, and Propositions 4.5, 4.6 and 4.7;

- the case of algebraic groups over $p$-adic fields (and $\mathrm{T}(d, k)$ for any local field), treated in the last subsection.

Let us now briefly explain how to deduce Theorem 6. Recall that every connected amenable Lie group has a closed, connected, cocompact, normal solvable subgroup (namely its solvable radical). Moreover, by a result of Mostow [20], any closed compactly generated subgroup $G$ of a solvable connected Lie group has a normal compact subgroup $K$, such that $G / K$ embeds as cocompact (hence nondistorted) subgroup of a solvable connected Lie group.

\subsection{Følner pairs and isoperimetric profile}

The notion of Følner pairs was introduced in [3] in order to produce lower bounds on the probability of return of symmetric random walks on graphs (or of the heat kernel on a Riemannian manifold). In [29], we defined a slightly different notion, called controlled Følner pairs.

\footnotetext{
${ }^{5}$ To see this, observe that if $g \in G, q \in Q$ and $h \in H$, then $g \eta(q) h=\eta\left(q^{\prime}\right) t h$, where $q^{\prime}=\bar{g} q \in Q$, and $t=\eta\left(q^{\prime}\right)^{-1} s \eta(q) \in H$.
} 
Definition 5.1. Let $G$ be a locally compact compactly generated group and let $S$ be a compact symmetric generating subset of $G$. A sequence $\left(F_{n}, F_{n}^{\prime}\right)$ of pairs of compact subsets is a sequence of controlled Følner pairs if there is a constant $C<\infty$ such that, for all $n \in \mathbb{N}$,

- $\mu\left(F_{n}^{\prime}\right) \leq C \mu\left(F_{n}\right)$;

- $S^{n} F_{n} \subset F_{n}^{\prime}$.

We will need the following easy fact:

Proposition 5.2 (Proposition 4.9 in [29]). If G has a sequence of controlled Følner pairs, then for all $1 \leq p \leq \infty, J_{G, p}^{b}(t) \succeq t$. Moreover, if $G$ is unimodular, then $j_{G, p}(t) \succeq \log t$.

\subsection{Algebraic groups over a local non-archimedean field}

Recall that an algebraic group over a p-adic field is isomorphic to a semidirect product $U \rtimes T$, where $T \simeq\left(k^{*}\right)^{d}$ acts semisimply on the unipotent radical $U$. Moreover, $G$ is compactly generated if and only if this action is non-degenerate in the sense that the weights of the action of $T$ on $G /[G, G]$ are nonzero (see Theorem 13.4 in [5] or Theorem 3.2.3 in [1]). If $x$ is a uniformizer in $k$, the cyclic subgroup generated by $x$ is a discrete and cocompact in $k^{*}$. Hence $G$ has a normal, cocompact closed subgroup of the form $U \rtimes \mathbb{Z}^{d}$.

Theorem 5.3. Let $U=U_{1} \times U_{2} \cdots$ be a finite direct product of algebraic nilpotent groups over local fields, and let $G=U \rtimes \mathbb{Z}^{d}$ be such that the action of $\mathbb{Z}^{d}$ on each $U_{i} /\left[U_{i}, U_{i}\right]$ is linear and has only nonzero weights. Then $G$ has a controlled sequence of Følner pairs. In particular (see Proposition 5.2), it satisfies $J_{G, p}^{b}(t) \approx t$

Proof. The idea of the construction is the following: $F_{n}$ (respectively, $F_{n}^{\prime}$ ) will be a product of a ball of radius $n$ (respectively, $2 n$ ) in $\mathbb{Z}^{d}$ with a certain compact subset of $U$. This latter subset will itself be a product of subsets of the $U_{i}$ 's. If the field $k_{i}$ is archimedean (see [29] for this case) then this subset will be a ball of $U_{i}$ of radius $a^{n}$ (resp. $b^{n}$ with $b>>a>1$ ), whereas if $k_{i}$ is non-archimedean, we will take the compact subgroup generated by all elements of $U_{i}$ of size (in $G$ ) at most $C n$ with $C$ large enough.

For the sake of concreteness, we will write the proof in the special case where $G=\mathrm{T}(d, k)$ is the group of invertible upper triangular matrices of size $d$ with coefficients in a non-archimedean local field $k$ (since again, the case of archimedean $k$ is treated in [29]).

Let us assume that $d \geq 2$ (the case $d=1$ being trivial). Let $v$ be a discrete valuation on $k$ (with values in $\mathbb{Z}$ ), and for every $x \in k$, let $|x|=e^{-v(x)}$ be the corresponding norm. We have

$$
|x+y| \leq \max \{|x|,|y|\} \quad \text { and } \quad|x y|=|x||y|
$$


Let $k_{n}$ be the (compact) subring of $k$ consisting of elements $y \in k$ of norm $|y| \leq e^{n}$. We fix a uniformizer $x_{0} \in k$ (i.e., such that $v\left(x_{0}\right)=-1$ ). We have

$$
x_{0} k_{n}=k_{n+1} \text {. }
$$

Let $U$ be the subgroup of $G$ consisting of unipotent elements, and let $T \simeq\left(k^{*}\right)^{d}$ be the subgroup of diagonal elements. We have a semidirect product

$$
G=T \ltimes U .
$$

For every $n \in \mathbb{N}$, let $U_{n}$ be the compact normal subgroup of $U$ consisting of unipotent matrices such that for $1 \leq i<j \leq d$, the $(i, j)$-entry lies in $k_{(j-i) n}$. We also consider the compact subset $T_{n}$ of $T$ defined by diagonal matrices whose diagonal entries and inverses have norms less or equal than $e^{n}$.

Let us identify $G$ with the cartesian product $T \times U$, where the group law is given by

$$
(t, u)(s, v)=\left(t s, u^{s} v\right)
$$

where $u^{s}=s^{-1} u s$. We define a compact subset $S$ of $G$ by

$$
S=T_{1} \cup U_{0} .
$$

Let $t \in T_{1}$. An easy computation shows that, for every $n \in \mathbb{N}$,

$$
t^{-1} U_{n} t \subset U_{n+1} \text {. }
$$

Moreover, $t_{0}=\left(x_{0}^{d-1}, x_{0}^{d-2}, \ldots, 1\right) \in T_{d-1}$ satisfies, for every $n \in \mathbb{N}$,

$$
t_{0}^{-1} U_{n} t_{0} \subset U_{n+1} \text {. }
$$

Note that since $G=\bigcup_{n} T_{n} \times U_{n}$, this implies that $S$ is a generating subset of $G$. On the other hand, we deduce from (5.2) that

$$
U_{n} \subset S^{2 d n+1} \text {. }
$$

As $T_{n} \subset S^{n}$, we have

$$
T_{n} \times U_{n} \subset S^{2 d n+n+1} .
$$

Claim 5.4. For all $n \geq 1, S^{n} \subset T_{n} \times U_{n}$.

Proof. This is true for $n=1$. Now, assume that this is true for $n \geq 1$, and take an element $g=(t, u)$ in $T_{n} \times U_{n}$, and an element $h$ of $S$. Let us check that $g h \in T_{n+1} \times U_{n+1}$. First, assume that $h=(s, 1) \in T_{1}$. Then,

$$
g h=\left(t s, u^{s}\right) \in T_{n+1} \times s^{-1} U_{n} s^{-1}
$$

By (5.1), $T_{n+1} \times s^{-1} U_{n} s^{-1} \subset T_{n+1} \times U_{n+1}$. Now, if $h=(1, v) \in U_{0}$, then $g h=$ $(t, u v) \subset T_{n} \times U_{n}$.

Now, let $F_{n}=T_{n} \times U_{2 n}$ and $F_{n}^{\prime}=T_{2 n} \times U_{2 n}$. We claim that $\left(F_{n}, F_{n}^{\prime}\right)$ is a sequence of Følner pairs. As $F_{n}^{\prime} \subset S^{4 d n+2 n+1}$ and $\left|F_{n}^{\prime}\right|=2\left|F_{n}\right|$, we just need to check that $S^{n} F_{n} \subset F_{n}^{\prime}$. Let $g=(t, u) \in S^{n} \subset T_{n} \times U_{n}$ and $g^{\prime}=(s, v) \in F_{n}$. By an immediate induction, (5.1) implies that $s^{-1} U_{n} s^{n} \subset U_{2 n}$. Hence,

$$
(t, u)(s, v)=\left(t s, u^{s} v\right) \in T_{2 n} \times U_{2 n} U_{n}=T_{2 n} \times U_{2 n},
$$

which finishes the proof. 


\section{Random walks}

We will prove a slightly more general result than the one stated in the introduction. Let $G$ be a locally compact, compactly generated group and let $S$ be a compact generating set of $G$. Let $(X, \mu, d)$ be a measure space, equipped with a measurable metric, on which $G$ acts measurably by isometries, and such that for every $x \in X$ the orbit map from $G$ to $X: g \rightarrow g x$ is a large-scale equivalence. If $X$ satisfies these assumptions, we will call it a geometric $G$-space. A typical example is if $G$ acts continuously, properly, and cocompactly on $X$.

Let $x_{0}$ be a point in $X$, and $r_{0}$ be such that every $x \in X$ lies at distance $<r_{0}$ from the orbit $G x_{0}$.

For every $x \in X$, let $\nu_{x}$ be a probability measure on $X$ which is absolutely continuous with respect to $\mu$. We assume moerover that

- (bounded support) there exists $r_{1}>0$ such that $\nu_{x}$ is supported in $B\left(x, r_{1}\right)$ for all $x \in X$,

- (non-degeneracy) there exists $c>0$ such that $p_{x}(y):=d \nu_{x} / d \mu(y) \geq c$ for all $y \in S B\left(x, r_{0}\right)$.

Denote by $P$ the Markov operator on $L^{2}(X)$ defined by

$$
P f(x)=\int f(g y) d \nu_{x}(y)
$$

Definition 6.1. Under the previous assumptions, we call $(X, P)$ a geometric $G$-random walk. Moreover, if $P$ is self-adjoint, then $(X, P)$ is called a symmetric geometric $G$-random walk.

Let $d P_{x}(y)=d \nu_{x}(y)=p_{x}(y) d \mu(y)$ and $d P_{x}^{n}(y)=p_{x}^{n}(y) d \mu(y)$.

Theorem 6.2. Let $G$ be a unimodular elementary solvable group with exponential growth. Then for every symmetric geometric $G$-random walk $(X, P)$, we have

$$
\sup _{x \in X} p_{x}^{n}(x) \approx e^{-n^{1 / 3}} .
$$

Proof. Since $X$ and $G$ are large-scale equivalent, Theorem 2.12 implies that their large-scale isoperimetric profiles are asymptotically equivalent. Therefore $j_{X, 2} \approx$ $\log t$. Theorem 2.7 then implies that the probability of return of any reversible random walk at large enough scale, decreases like $e^{-n^{1 / 3}}$. To apply this to our random walk $P$, we just need to check that for $k$ large enough, $P^{k}$ satisfies the conditions (i) to (iii) of Section 2.6, with $h$ as big as we want. This follows easily from the definition of $r_{0}, r_{1}$ and $c$, and the fact that $S$ generates $G$.

\section{References}

[1] Abels, H.: Finite presentability of S-arithmetic groups. Compact presentability of solvable groups. Lecture Notes in Mathematics 1261, Springer-Verlag, Berlin, 1987.

[2] Alexopoulos, G.A.: Lower estimate for central probabilities on polycyclic groups. Canad. J. Math. 44 (1992), no. 5, 897-910. 
[3] Barlow, M., Coulhon, T., And Grigor'yan, A: Manifolds and graphs with slow heat kernel decay. Invent. Math. 144 (2001), no. 3, 609-649.

[4] Bekka, B., de la Harpe, P., And Valette, A.: Kazhdan's Property (T). New Mathematical Monographs 11, Cambridge University Press, Cambridge, 2008.

[5] Borel, A. And Tits, J.: Groupes réductifs. Inst. Hautes Études Sci. Publ. Math. 27 (1965), 55-152.

[6] Coulhon, C.: Espaces de Lipschitz et inégalités de Poincaré. J. Funct. Anal. 134 (1996), no. 1, 81-113.

[7] Coulhon, C.: Random walks and geometry on infinite graphs. In Lecture notes on analysis on metric spaces (Trento, 1999), 5-36. Appunti Corsi Tenuti Docenti Sc., Scuola Norm. Sup., Pisa, 2000.

[8] Cheeger, A. And Gromov, M.: $L^{2}$-cohomology and group cohomology. Topology 25 (1986), no. 2, 189-215.

[9] Coulhon, T., Grigor'yan, A and Pittet, C.: A geometric approach to ondiagonal heat kernel lower bounds on groups. Ann. Inst. Fourier (Grenoble) 51 (2001), no. 6, 1763-1827.

[10] Coulhon, T. And Saloff-Coste, L.: Variétés riemanniennes isopérimétriques à l'infini. Rev. Mat. Iberoamericana 11 (1995), no. 3, 687-726.

[11] Coulhon, T. And Saloff-Coste, L.: Isopérimétrie pour les groupes et les variétés. Rev. Mat. Iberoamericana 9 (1993), no. 2, 293-314.

[12] Cornulier, Y., Tessera, R. And Valette, A.: Isometric group actions on Hilbert spaces: growth of cocycles. Geom. Funct. Anal. 17 (2007), no. 3, 770-792.

[13] Dioubina, A.: Instability of the virtual solvability and the property of being virtualy torsion free for quasi-isometric groups. Internat. Math. Res. Notices 21 (2000), 1097-1101.

[14] Erschler, A.: On isoperimetric profiles of finitely generated groups. Geom. Dedicata 100 (2003), 157-171.

[15] Gromov, M.: Asymptotic invariants of infinite groups. In Geometric group theory, Vol. 2 (Sussex, 1991), 1-295. London Math. Soc. Lecture Note Ser. 182, Cambridge Univ. Press, Cambridge, 1993.

[16] Hall, P.: The Frattini subgroups of finitely generated groups. Proc. London Math. Soc. (3) 11 (1961), 327-352.

[17] Hebisch, P.: On heat kernels on Lie groups. Math. Z. 210 (1992), no. 4, 593-605.

[18] Hebisch, P. And Saloff-Coste, L.: Gaussian estimates for Markov chains and random walks on groups. Ann. Probab. 21 (1993), no. 2, 673-709.

[19] Kanai, M.: Rough isometries, and combinatorial approximations of geometries of non-compact Riemannian manifolds. J. Math. Soc. Japan. 37 (1985), no. 3, 391-413.

[20] Mostow, G.D.: Some applications of representative functions to solvmanifolds. Amer. J. Math. 93 (1971), 11-32.

[21] Mustapha, S.: Distorsion des distances dans les groupes p-adiques. Bull. Sci. Math. 124 (2000), no. 3, 175-191.

[22] Mustapha, S.: Random walks on unimodular p-adic groups.. Stochastic Process. Appl. 115 (2005), no. 6, 927-937.

[23] Pittet, C.: Følner sequences in polycyclic groups. Rev. Mat. Iberoamericana 11 (1995), no. 3, 675-686. 
[24] Pittet, C.: The isoperimetric profile of homogeneous Riemannian manifolds. J. Differential Geom. 54 (2000), no. 2, 255-302.

[25] Pittet, C. And Saloff-Coste, L.: Random walks on finite rank solvable groups. J. Eur. Math. Soc. 5 (2003), no. 4, 313-342.

[26] Pittet, C. and Saloff-Coste, L.: Random walks and isoperimetry on discrete groups of Lie groups. In Random walks and discrete potential theory (Cortona, 1997), 306-319. Sympos. Math. 39, Cambridge Univ. Press, Cambridge, 1999.

[27] Roe, J.: Lectures on coarse geometry. University Lecture Series 31, American Mathematical Society, Providence, RI, 2003.

[28] Tessera, R.: Asymptotic isoperimetry of balls in metric measure spaces. Publ. Mat. 50 (2006), no. 2, 315-348.

[29] Tessera, R.: Asymptotic isoperimetry on groups and uniform embeddings into Banach spaces. Comment. Math. Helv. 86 (2011), no. 3, 499-535.

[30] Tessera, R.: Vanishing of the first reduced cohomology with values in a $L^{p}$-representation. Ann. Inst. Fourier (Grenoble) 59 (2009), no. 2, 851-876.

[31] Tessera, R.: Large scale Sobolev inequalities on metric measure spaces and applications. Rev. Mat. Iberoam. 24 (2008), no. 3, 825-864.

[32] Varopoulos, N.: Isoperimetric inequalities and Markov chains. J. Funct. Anal. 63 (1985), no. 2, 215-252.

[33] Varopoulos, N.: Long range estimates for Markov chains. Bull. Sci. Math. (2) 109 (1985), no. 3, 225-252.

[34] Varopoulos, N.: Théorie du potentiel sur des groupes et des variétés. C. R. Acad. Sci. Paris Sér. I Math. 302 (1986), no. 6, 203-205.

[35] Varopoulos, N.: Convolution powers on locally compact groups. Bull. Sci. Math. (2) 111 (1987), no. 4, 333-342.

[36] Varopoulos, N.: Analysis on Lie groups. J. Funct. Anal. 76 (1988), no. 2, 346-410.

[37] Varopoulos, N.: Small time Gaussian estimates of heat diffusion kernels. II. The theory of large deviations. J. Funct. Anal. 93 (1990), no. 1, 1-33.

[38] Varopoulos, N.: Analysis and geometry on groups. In Proceedings of the International Congress of Mathematics, Vol I, II (Kyoto, 1990), 951-957. Math. Soc. Japan, Tokyo, 1991.

[39] Varopoulos, N.: Groups of super polynomial growth. In Proc. ICM Sattelite Conf. on Harmonic Anal., 194-200. Springer, Berlin, 1991.

[40] Varopoulos, N., Saloff-Coste, L. and Coulhon, T.: Analysis and geometry on groups. Cambridge Tracts in Mathematics 100, Cambridge University Press, Cambridge, 1992.

[41] WeIL, A.: Basic number theory. Classics in Mathematics, Springer-Verlag, Berlin, 1995.

Received August 12, 2011.

Romain Tessera: UMPA, ENS Lyon, 46 Allée d'Italie, 69364 Lyon Cedex, France.

E-mail: romain.tessera@ens-lyon.fr 